\title{
Microscopylnnovations
}

\section{Microscopy Today Innovation Awards}

The editors of Microscopy Today congratulate the winners of the eighth Microscopy Today Innovation Award competition. The ten innovations described below advance microscopy in several areas: light microscopy, scanning probe microscopy, and electron microscopy. These innovations will make microscopy and microanalysis more powerful, more flexible, more productive, and easier to accomplish.

\section{Black-Silicon Induced-Junction Silicon Photodiode}

\section{Aalto University, Finland}

Developers: Mikko Juntunen, Juha Heinonen, Ville Vähänissi, Päivikki Repo, and Hele Savin

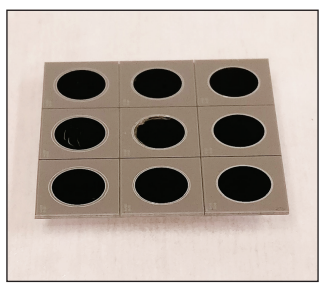

This new photodiode eliminates the front surface reflectance problem through a nanostructure created via inductively coupled plasma-reactive ion etching (ICP-RIE). The resulting "black silicon" nanostructure exhibits feature sizes smaller than the wavelength of visible light. The structure creates a refraction index gradient, which causes essentially all incident photons to be absorbed, eliminating front surface reflectance for a broad wavelength range (250-950 $\mathrm{nm}$ ) and delivering $96 \%$ quantum efficiency up to incident angles of $70^{\circ}$. The nanostructure also diffracts light, increasing its optical path and increasing the response of these diodes in the near-infrared range.

To eliminate recombination losses caused by implantation and dopants, a 20-nanometer-thick, negatively charged, alumina $\left(\mathrm{Al}_{2} \mathrm{O}_{3}\right)$ film is deposited on top of the nanostructure. The negative charge repels electrons and attracts holes, creating an inversion layer at the surface, resulting in an induced junction. The induced junction causes no Auger recombination and no lattice damage, ensuring high collection efficiency of low-wavelength photons absorbed close to the surface. The wavelength regime around $250-300 \mathrm{~nm}$ has been a particular issue for commercial silicon photodiodes, and their quantum efficiency has been limited to around 50\% in this region. In contrast, this new photodiode surpasses even a $100 \%$ quantum efficiency around $300 \mathrm{~nm}$, as the excited electrons gain enough energy to ionize secondary electrons via impact ionization. Aside from better response, the new device exhibits performance similar to commercial photodiodes with respect to other figures of merit, like speed and linearity. The manufacturing process for these diodes has fewer high-temperature steps than the typical commercial silicon photodiode process, which drives down production costs.
These new photodiodes are expected to be most useful where commercial diodes perform the worst: UV spectroscopy, UV light detection, and near-infrared broadband applications in low-light imaging. Additionally, these diodes should be important when sensing diffuse light, as when detecting X-rays or gamma-rays with scintillators coupled to photodiodes. In such applications, the high-energy photon is absorbed by a scintillator material, which then in turn emits photons of visible wavelengths. Because a significant fraction of the secondary photons exits the scintillator at a high angle, the improved performance of the new diode at high incident angles should be useful.

\section{NanoScratch In-Situ Nanomechanical Testing in the SEM}

\section{Bruker-Hysitron}

Developers: Jason Oh, Syed Asif, Bartosz Nowakowski, Ryan Major, Sanjit Bhowmick, Eric Hintsala, Doug Crowson, Bernie Becker, and Edward Cyrankowski

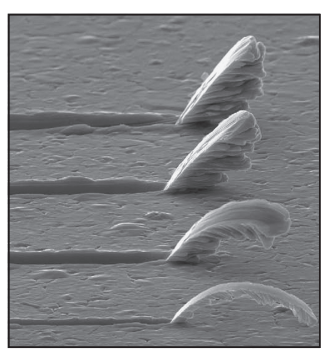

The Hysitron PI 88 is a depthsensing nanomechanical test instrument designed for use inside scanning electron microscopes (SEMs). This stage-mounted instrument provides quantitative microand nano-scale mechanical property measurements synchronized with SEM imaging. The nanoScratch option for the PI 88 enables quantitative measurement of both normal and lateral forces during in-situ nanomechanical tests, providing tribological information such as friction coefficient and delamination force. Reciprocating scratch tests can also be performed to study wear mechanisms over longer periods of time. Acquired data provide information concerning material behavior under simultaneous normal and lateral stresses, which may be supplemented by simultaneous high-resolution SEM imaging of the deformation process.

Traditional in-situ nano- and micro-scale mechanical testing is conducted by applying stress and strain to a sample in the normal direction to obtain hardness and modulus through targeted nanoindentation testing or yield behavior through small-scale compression or tensile tests. However, the uniaxial nature of the tests makes it difficult to quantify material behavior under sliding conditions.

With nanoScratch, in-situ tribological tests are accomplished by applying a normal load to the sample while also moving the sample laterally at a prescribed rate. During the test, force and displacement are measured between the probe and sample in both the normal and lateral directions using sensitive capacitive transducers. The user-changeable probe is 


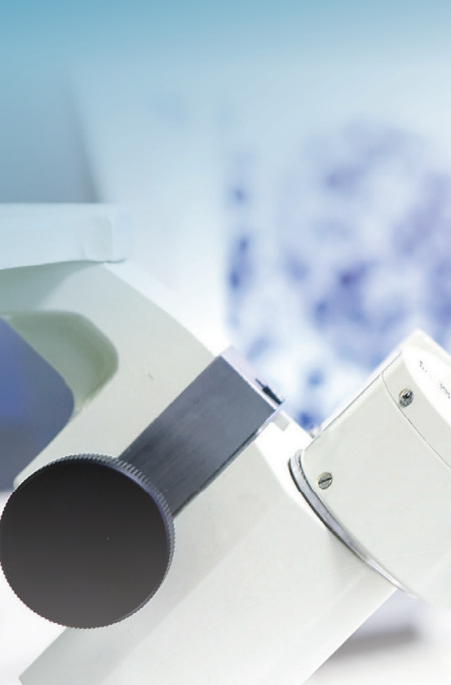

ARGYLE DIAMOND PARTNER

OPAL PARTNERS

GOLD PARTNERS

\section{ThermoFisher S C I EN TIFIC}
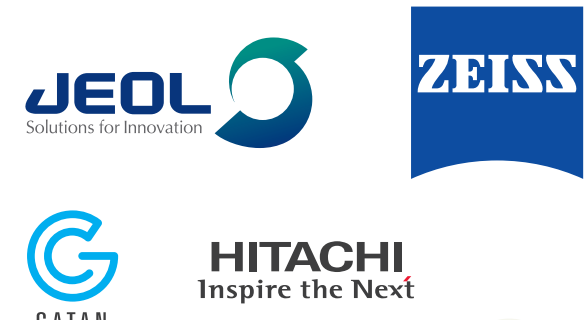

GATAN
HITACHI

Inspire the Next

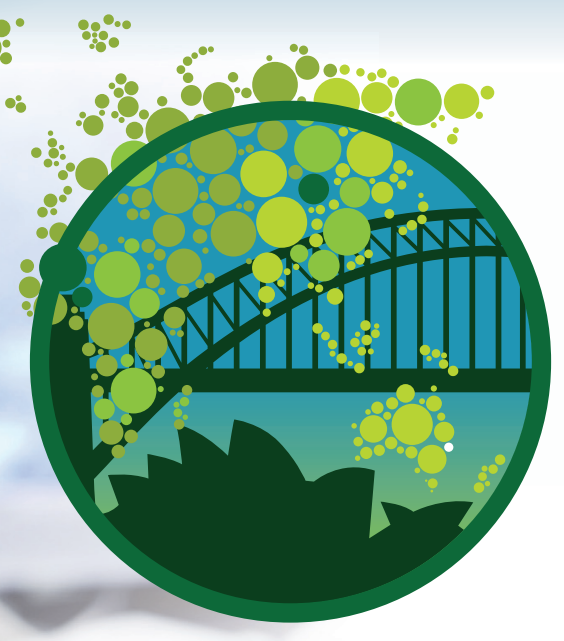

Join us at the

19TH INTERNATIONAL MICROSCOPY CONGRESS
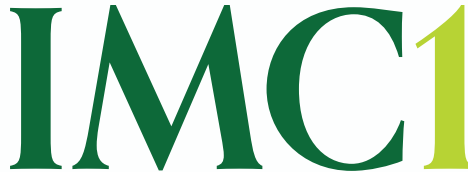

Sydney 9-14 September 2018 International Convention Centre

\section{BRIDGING THE SCIENCES}

\section{WHY ATTEND IMC19?}

- Be part of an outstanding scientific program led by world-renowned plenary speakers

- Network and share experiences and ideas with over 2000 colleagues and peers from across the globe

- Keep up to date with the latest advancements and developments in microscopy and visit our interactive trade exhibition

- Experience the stunning new International Convention Centre right in the heart of Sydney

\section{KEY DATES}

Call for Abstracts - 1 November 2017

Position your brand at the frontline with other global leaders in microscopy.

Be represented at the premier global microscopy Congress. IMC19 sponsors and exhibitors will achieve superior brand exposure and direct networking opportunities with professionals from all disciplines of microscopy.

For more information or a copy of the Prospectus, contact Drew Whait on:

Email: sponsorship@arinex.com.au

Phone: +61292650700
Registration Opens - 1 November 2017 Abstract Submission Deadline - 1 February 2018 Early Bird Registration Closes - 10 May 2018 Author Registration Deadline - 10 May 2018

Download our Program at a Glance at wwwimc19.com

\section{VISIT SYDNEY -} A WORLD CLASS DESTINATION

Sydney is renowned for its stunning harbour setting, temperate climate, and dining scenes. Whilst at IMC19 explore the iconic Opera House and

Harbour Bridge and experience a myriad of other unique Australian experiences.
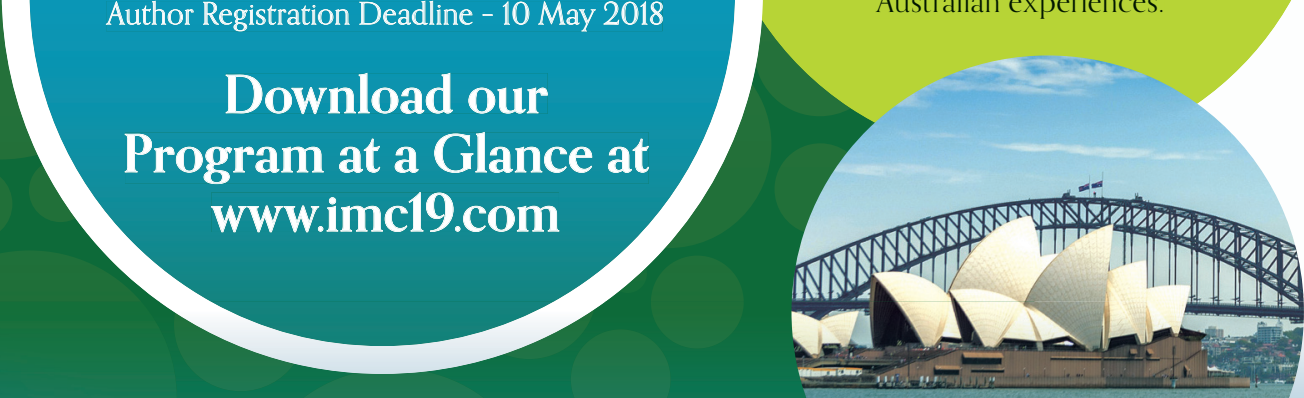
typically diamond. Lateral sample movement is accomplished through a long-range piezoelectric actuator, and the lateral velocity and maximum distance can be varied to replicate a variety of sliding conditions. Since these tests are inside an SEM, the probe-sample contact can be precisely controlled to ensure proper test placement. Real-time observation of sample deformation can be recorded and ultimately correlated to the force and displacement data acquired by the PI 88 . The lateral force sensor is capable of measuring small-scale forces between $3-30,000 \mu \mathrm{N}$, and the maximum lateral actuation distance is $30 \mu \mathrm{m}$.

One application for this development is quantitative scratch testing of thin films and coatings for determining interfacial strength. For such applications cracking, buckling, delamination, and spallation events observed with the SEM imaging can, for the first time, be directly correlated to the force and displacement data.

\section{Keyence VR-3000 Non-Contact Wide-Area 3D Measurement System}

\section{Keyence Corporation of America}

\section{Developers: Keyence Corporation of America}

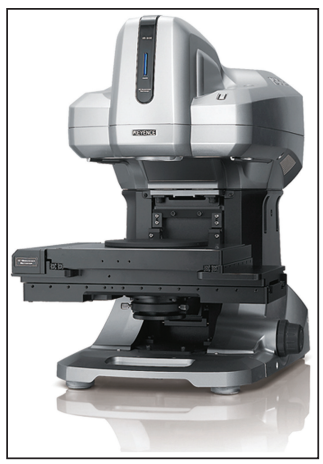

The VR-3000 is a non-contact, wide-area $3 \mathrm{D}$ measurement microscope that performs sub-micron resolution scanning in seconds. After scanning, the microscope creates a 3D model of the sample, which can be analyzed for form, contour, flatness, warpage, curvature, line roughness (ISO 4287:1997), surface roughness (ISO 25178), and more. Scans can be directly overlaid against 3D computer-aided design (CAD) models to visualize and quantify surface shape differences, and pass/fail inspection can be programmed to save time in quality and analysis applications such as setting prototype conditions or performing acceptance inspections.

Structured light is emitted from the transmitter lens and projected onto the surface of the sample. When the reflected light is viewed from a different angle under the receiver lens, the light appears banded and bent due to height changes on the object's surface. A CMOS camera is used to capture the reflected light, and based on triangulation the height and position can be calculated using a telecentric multi-triangulation algorithm in about 4 seconds. The system is able to scan up to a 4 " $\times 8$ " envelope size, and the optics can be mounted on a gantry to accommodate larger applications.

Traditionally, probe-based profilers have been the industry standard for analyzing 3D profiles and surface shapes. However, there are important disadvantages to probe-based profiles. Probe-based profilers use a stylus or probe-tip to trace the surface of a sample, which means only a single line of data can be obtained. Styluses must make contact with the sample surface, and scratches may form on the surface of a sample.
Also soft or viscous samples are difficult to measure with probe-based profilers.

The VR-3000 uses a non-contact white light as its measurement medium, which means that it can overcome all the disadvantages of probe-based profilers. Additionally, the VR-3000 provides the analysis capabilities of 3D CAD compare, 3D CAD export of scans, and XYZ traceability. Applications can be found in any industry that requires $3 \mathrm{D}$ surface characterization and quantification, including but not limited to automotive and tooling (brake pads, tooling, gears, ductile metal fractures), electrical components (ball grid arrays, connector pins, printed circuit boards), injection molding, and many others.

\section{Plasmonic Grating Platform to Replace Glass Slides}

\section{University of Missouri}

Developers: Shubhra Gangopadhyay, Keshab Gangopadhyay, Aaron Wood, Biyan Chen, Sangho Bok, Haisheng Zheng, and Joseph Mathai

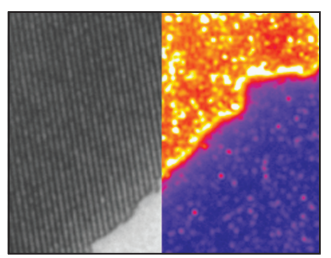

Plasmonic gratings can improve signal-to-noise and image resolution in light microscopy. Nanoscale plasmonic gratings use incident light photons at specific angles and wavelengths to excite electron oscillations at the surface in a process known as surface plasmon resonance (SPR). Previously, SPR-based imaging platforms were expensive to fabricate and required advanced optical systems to excite and image. The present system employs a scalable glancing angle deposition (GLAD) technique to produce an abundance of nanoscale silver protrusions from the silver grating surface that concentrate the surrounding electromagnetic field formed during SPR. In the GLAD technique, a silver metal film is thermally evaporated at a "glancing" angle onto a grating surface, and the process is tuned to produce a dense population of nano-protrustions over the entire grating surface. When this process is combined with a microcontact lithography process, inexpensive GLAD plasmonic gratings can be fabricated. These gratings can be used as a substitute for quartz and glass microscopy slides to improve the signal-to-noise ratio (SNR) and image resolution in light microscopy.

A plasmonic grating also may be tuned to couple incident light in the visible spectrum provided by a microscope objective. When fluorescent molecules, such as those used to label proteins, are located near these nano-protrusions, they interact in a manner that greatly enhances the number of emitted photons and ultimately results in a higher emission of intensity detected from individual molecules without an increase in background intensity. The plasmonic grating and nano-protrusions also have far-field superlensing capabilities, which transmit the near-field information into the farfield to improve the observed image resolution. 
The GLAD plasmonic grating platform can improve the SNR and precision of current localization microscopy techniques such as stochastic optical reconstruction microscopy (STORM) and photo-activated localization microscopy (PALM). This enables the imaging of a wider range of fluorophore concentrations. The nano-protrusions on GLAD gratings enable simultaneous imaging of large sample areas without using point-by-point scanning techniques, reducing image collection time and minimizing photobleaching. The enhancement provided by the GLAD grating also enables the imaging of single-molecule fluorescence with less expensive systems such as the simple epifluorescence microscopes available in most labs.

\section{Scanning Helium Microscope (SHeM)}

\section{University of Newcastle, Australia}

\section{Developer: Paul Dastoor}

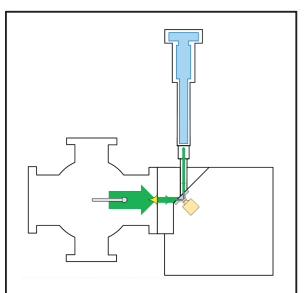

Scanning helium microscopy (SHeM) is based on helium atom scattering (HAS), an established technique for investigating surface structure. In a typical HAS experiment, monochromatic, very-low-energy (10-300 meV) helium atoms are scattered from a surface, under vacuum conditions, and detected in a tuned mass spectrometer. HAS is a sensitive, non-perturbing probe of surface morphology that can be used to analyze metals, insulators, semiconductors, and organic materials. The SHeM operates analogously to a scanning electron microscope (SEM) but with the electron beam replaced with a beam of neutral helium atoms. The beam of helium atoms (created via free-jet expansion in the source chamber) emerges through a small pinhole to strike the sample surface, and reflected helium atoms are collected in the detector chamber. By rastering the sample back and forth, an image of the surface is constructed.

The very-low-energy (a few $\mathrm{meV}$ ) neutral helium atoms cannot penetrate the surface at all, but rather they backscatter from the outermost electronic corrugation of the sample, giving the technique absolute surface sensitivity. The SHeM is able to exploit both electron-atom (elasticscattering) and electron-phonon-atom (inelastic-scattering) interactions to deliver both topological and chemical contrast simultaneously. Moreover, the chemical sensitivity is still apparent even under several atomic layers of adsorbed contaminant.

A range of material systems remains difficult to image with conventional light and electron microscopies: transparent, fragile, weakly-bonded, insulating, very rough, and magnetic samples. These delicate structures (such as biological samples, organic films for polymer electronics, and adsorbate layers) suffer degradation under the energetic probes of traditional microscopies. Furthermore, the charged nature of certain probes presents difficulties when imaging insulating materials without the addition of a conductive coating. SHeM is able to image such structures completely non-destructively since the neutral helium beam is a chemically, electrically, and magnetically inert probe of the sample surface. Applications of SHeM include the investigation of new explosives and the imaging of organic electronic materials and circuits in operando without damaging or influencing the operation of the circuitry. Thus, the SHeM enables a wide range of surfaces to be studied for the first time.

\section{STEM Method for Observing Magnetic Ordering with Atomic Resolution}

Oak Ridge National Laboratory and University of Uppsala, Sweden

Developers: Juan Carlos Idrobo and Ján Rusz

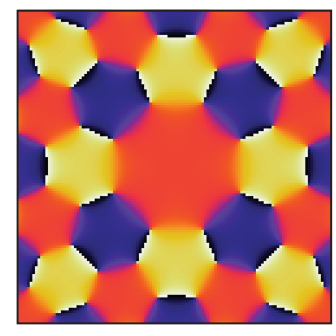

Energy-loss (or electron) magnetic circular dichroism (EMCD) is a spectroscopic method used to measure the magnetic properties of materials in scanning transmission electron microscopy (STEM). Until now, classical EMCD measurements were performed either in diffraction mode or by using small convergenceangle electron probes. These two approaches intrinsically limit the spatial resolution of the measurements. A recent experiment demonstrated that the poor spatial resolution of previous EMCD measurements can be overcome by using aberrated electron probes. In recent years, much effort has been directed toward correcting aberrations in electron beams to achieve high-resolution imaging and analysis. Rather than eliminating the aberrations in the electron microscope to achieve a smaller probe size, this new method intentionally introduces a non-cylindrical aberration and then uses this aberrated electron probe to acquire atomically resolved electron energy-loss spectra. The acquired spectra then can be analyzed to reveal the magnetic ordering of a material. The original work used a four-fold astigmatism aberration (because the material had four-fold crystal symmetry), but different aberrations can be selected depending on the crystal symmetry of the material. This "aberrated-probe method" is based on electron energyloss spectrometry, and thus the technique also reveals the chemical nature of a magnetic phase, which is not achievable via Lorentz microscopy.

The new method demonstrates that phases in electron probes can be used in ways analogous to polarization of light and X-rays. Neither light nor X-ray synchrotrons, however, can achieve the spatial resolution of an electron microscope, and therefore electron microscopy has a special capability for studying chiral properties of materials at atomic resolution. This new analysis method allows the study of magnetic phases in materials at a new level of precision. For instance, magnetic domains in magnetic storage hard drives could be investigated at the atomic level. The method is not 
limited to magnetic dichroism but can be applied to other chiral signals such as optical dichroism, valley polarization, skirmions, complex topological vortex electrical polarization, and other novel states of matter. The new technique also could provide a mechanistic understanding of the behavior of magnetic nanoparticles under investigation for gene and drug delivery.

\section{Livecyte ${ }^{\circledR}$ Kinetic Cytometer}

\section{Phasefocus}

Developers: Martin Humphry, Kathryn Cooper, Tim Godden, Richard Kasprowicz, Bhupalreddy Kumbhum, Kevin Langley, Andrew O’Brien, James Russell, Chris Shuttle, Matthew Stagg, Rakesh Suman, and Joanne Whetstone

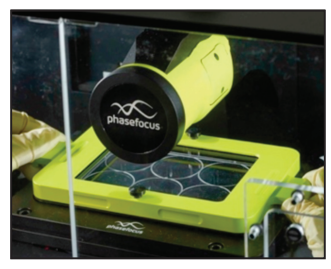

The Livecyte Kinetic Cytometer uses ptychographic quantitative phase imaging (QPI) microscopy to measure and analyze the behavior of single or multiple live cell types, on a cell-by-cell basis and on a population basis, in long time-lapse sequences typically lasting many hours or days. Culture samples are prepared by the user in industry-standard micro-well plates and studied within an integral incubator that maintains optimal temperature, humidity, and carbon dioxide levels. A custom-designed "Sample Pod" protects cells during transport from the user's laboratory into the system and permits perfect realignment if the cells need to be removed temporarily during an experiment (for example to add a drug treatment or replace culture media). In QPI, images with very high inherent contrast are generated as a result of phase delays introduced as the illuminating light passes through the cells, eliminating the necessity for exogenous stains or labels.

Intuitive graphical user interface tools may be used to program fully automated ptychographical time-lapse workflows interspersed (when desired) with conventional fluorescence imaging. The combination of ptychography and fluorescence imaging enables special measurement protocols such as the extraction of single-cell or cell-population kinetic phenotypes in parallel with fluorescence-based functional studies. Imaging may be performed continuously and automatically over multiple regions of interest within single or multiple wells. Livecyte's Cell Analysis Toolbox software provides automated cell tracking that can track hundreds of cells on an individual basis. Typical automated assays and analyses include the following: cell motility, speed, and meandering index; cytotoxic response and cell viability including apoptosis rate; dynamic proliferation analysis including time-resolved mitotic index measurement; and morphological cell parameter measurements (dry mass, volume, sphericity, etc.).

Traditional fluorescence microscopes often employ high light intensities that can induce photobleaching and phototoxicity.
By comparison, ptychography's inherently high contrast, and Livecyte's illumination power thousands of times lower than fluorescence methods, offer an extremely "gentle" experimental environment.

Applications well suited for Livecyte include analysis of the phenotypic behavior of "closer to real life" patientderived primary cells and stem cells as they come under the influence of pharmaceutical drug candidates, including drugs that are uniquely toxic to cancer cells or, conversely, drugs that encourage cell behaviors associated with wound healing.

\section{Benthic Underwater Microscope}

\section{Scripps Institution of Oceanography, University of California at San Diego}

Developers: Andrew D. Mullen, Tali Treibitz, Paul L.D. Roberts, and Jules S. Jaffe

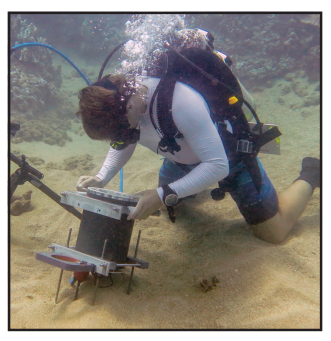

Biological processes occurring at microscopic scales significantly impact the health of important marine ecosystems, including coral reefs and kelp forests. It is critical to observe such processes in their natural settings because of the complex biological physical interactions occurring in the ocean environment. The benthic underwater microscope is the first instrument capable of acquiring microscopic images of seafloor organisms in situ, in the natural underwater environment. This diver-operated system allows non-invasive observations of live, threedimensional specimens at a resolution of $\sim 2 \mu \mathrm{m}$.

Unique challenges of underwater microscopy are overcome through the combination of three principal optical components: a long-working-distance microscope objective, an electrically tunable lens (ETL), and focused reflectance illumination. A long-working-distance objective lens provides the numerical aperture needed to resolve fine details, while also offering sufficient working distance to leave the subject undisturbed. Rapid focusing is achieved through the use of a shape-changing ETL; the curvature of this deformable lens can be controlled in order to modulate the system's focal length. This produces a compact means of bringing a subject into precise focus as well as providing the ability to scan through a volume, in order to collect focal stacks of three-dimensional subjects. Finally, a ring of focused LEDs provides high-intensity reflectance illumination, which allows the short exposures needed to prevent motion blur. The optical system is integrated into a submersible package that includes camera, electronics, and user interface. The system has been used to collect still images as well as videos and overnight time-series image sets showing organism behaviors. By allowing observations at important yet previously unattainable scales, the instrument provides a new window into the marine world. 
Previously, micro-scale observations of benthic marine specimens could only be performed on samples in the lab under a bench-top microscope. This significantly limited the types of processes that could be observed. Ocean habitats are complex settings, and many interactions occur between different species; additionally the organisms interact with a dynamic physical environment that has continuous fluctuations in parameters such as temperature, fluid motion, and $\mathrm{pH}$. This environment is thus exceedingly difficult to fully simulate in the lab.

\section{Scanning Thermo-Ionic Microscopy (STIM)}

\section{University of Washington and Shenzhen Institutes of Advanced Technology}

Developers: Jiangyu Li, Ahmadreza Eshghinejad, and Ehsan Nasr Esfahani

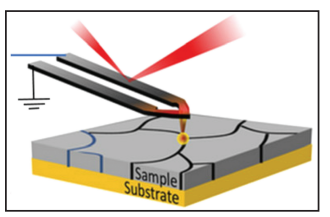

Scanning thermo-ionic microscopy (STIM) probes local electrochemical activities via Vegard strain induced by thermal stress. Vegard strain is a lattice volume change resulting from a change in the concentration of an ionic species. This strain can be produced by local temperature fluctuations induced either by resistive heating or photo-thermal heating, resulting in thermal expansion and a stress that drives ionic oscillation. The Vegard strain resulting from this ionic oscillation can then be detected at appropriate harmonics, enabling sensitive detection with high spatial resolution. This is the essence of STIM. The dynamics of ionic motion can be captured from point-wise spectroscopy studies, while spatial inhomogeneity can be revealed by STIM mapping.

Implementation of this new method has been demonstrated on modified commercial atomic force microscopes (AFMs). Detection of the harmonic responses of cantilever deflection, usually very small in magnitude, were accomplished by a lock-in amplifier around the cantilever-sample contact resonance frequency, which enhances the signal-to-noise ratio by orders of magnitude. To avoid topography cross-talk during STIM scanning, a dual amplitude resonance tracking (DART) technique was used to track the contact resonance.

Conventional electrochemical characterization techniques are very difficult to scale down because they are mostly based on current measurement, requiring the detection of small currents on the order of picoamperes at the nanoscale. An earlier scanning probe technique of electrochemical strain microscopy (ESM) offers high sensitivity and spatial resolution in exciting Vegard strain electrically. However, it is often difficult to distinguish Vegard strain from other electromechanical mechanisms such as the piezoelectric effect, electrostatic interactions, and capacitive forces. STIM overcomes this difficulty by using Vegard strain excited by stress instead of an electric field, and it still provides high sensitivity and spatial resolution similar to ESM. Since excitation is not electrical in nature-eliminating the interference from other electrochemical mechanisms-in operando testing becomes possible. The STIM method can be applied to study a wide range of electrochemical materials including electrode materials for battery systems, fuel cells and flow batteries, electrocatalysts for efficient electrosynthesis of liquid fuels, and photoelectrochemical materials that can directly convert solar energy to fuels.

\section{Multimodal Imaging with a Coherence Switching Laser}

\section{Yale University}

Developers: Sebastian Knitter, Changgeng Liu, Brandon Redding, Mustafa K. Khokha, Michael A. Choma, and Hui Cao

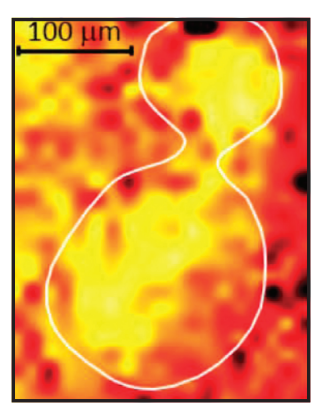

Unlike the traditional laser sources with a fixed degree of high spatial coherence, degenerate vertical external-cavity surface-emitting lasers (VECSELs) can have a tunable degree of spatial coherence, which is a new degree of freedom in microscopy. This new laser source can switch between emission of two different spatial coherence states: high spatial coherence and low spatial coherence. These two coherence states yield different kinds of imaging contrast in light microscopy, and the ability to perform coherence switching enables multimodal microscopic imaging. Low-spatial-coherence illumination enables speckle-free imaging of structures in specimens. High-spatialcoherence illumination enables dynamic speckle imaging of moving objects and blood flow in specimens. In particular, speckle fluctuation analysis yields metrics that are related to blood flow speed. This contrast is endogenous in nature and does not require the use of external contrast agents.

A degenerate laser has a self-imaging laser cavity; one end of the resonator cavity is imaged onto the other end of the cavity. By placing a wide-area gain element at one end of the cavity, the resonator supports a large number ( 1000) of non-overlapping, mutually incoherent modes. For our laser, a wide-area electricallypumped semiconductor VECSEL was used as the gain element. A large number of transverse lasing modes combine to generate lowspatial-coherence (speckle-free) emission. The use of a spatial filter within the self-imaging cavity can effectively phase-lock the large number of modes into a single or a few spatial modes while maintaining emission power and direction. A degenerate laser with a VECSEL gain element also has the advantages of relatively low cost and uncomplicated thermal management. Additionally, degenerate lasers are much brighter than traditional light sources used in microscopy (for example, thermal sources and LEDs).

The ability to perform multimodal imaging was demonstrated in a frog embryo (a tadpole, an important animal model in congenital heart disease research). Dynamic structural imaging of the embryo heart was performed using speckle-free, low-spatial-coherence illumination, whereas functional speckle flow imaging was accomplished with high-spatial-coherence illumination. This imaging demonstrated heart wall and blood flow dynamics over the cardiac cycle (that is, the heart filling with blood and the heart ejecting blood to the body). 